\title{
RADIATION CHOROIDAL VASCULOPATHY: AN INDOCYANINE GREEN ANGIOGRAPHY STUDY
}

\author{
W. M. K. AMOAKU, B. LAFAUT, G. SALLET and J. J. DE LAEY \\ Gent, Belgium
}

\begin{abstract}
SUMMARY
An indocyanine green angiography (ICG-A) study of choroidal vasculature in the irradiated peritumoral zone in 16 eyes treated with ${ }^{106} \mathrm{Ru} /{ }^{106} \mathrm{Rh}$ brachytherapy for choroidal melanoma was performed between 4 and 72 months following treatment. The earliest changes observed were peritumoral atrophy of the retinal pigment epithelium and choriocapillaries. With time, the larger choroidal vessels progressively became non-perfused. There was only minimal staining of the smaller vessels. However, at 2 years or more after radiotherapy the larger choroidal vessels showed progressive vascular wall staining with ICG ('perivasculitis') associated with continuing closure. Previous histopathological studies of radiation damage to the choroid have demonstrated atrophy and occlusion of the vasculature but have shown no evidence of vasculitis. It is suggested that ICG staining of the choroidal vascular wall in radiation choroidopathy is due to radiation-induced endothelial cell loss or dysfunction rather than a true vasculitis. Subsequent progression results in vascular occlusion, and development of new clusters of abnormal choroidal vessels.
\end{abstract}

The effects of ionising radiation on the chorioretina are more extensive and occur more often with brachytherapy than with teletherapy because of the much higher doses of radiation employed. In order to control choroidal melanoma, some overlap of normal tissue receives uniformly effective doses of radiation. ${ }^{1}$ This provides an opportunity to study the effects of ionising radiation on the normal peritumoral choroid, although the secondary effects of tumour and its products, and post-radiation tumour necrosis and retinal pigment epithelial (RPE) changes, may influence the radiation changes in the posterior segment.

From: Department of Ophthalmology, University Hospital Gent, de Pintelaan 185, B-9000 Gent, Belgium.

Correspondence to: W. M. K. Amoaku, Department of Ophthalmology, University of Nottingham, Queen's Medical Centre, Nottingham NG7 2UH, UK.
Fundus fluorescein angiograms (FFAs) after brachytherapy for choroidal melanoma show a gradually progressive occlusion of the choroidal vasculature $^{2-6}$ in the tumour and peritumoral area. The vascular changes in conjunction with direct toxicity of radiation contribute to tumour destruction. 7,8

Similarly, previous experimental FFA studies in the rabbit eye have shown choroidal vaso-occlusion after beta-irradiation with ruthenium-106/rhodium$106\left({ }^{106} \mathrm{Ru} /{ }^{106} \mathrm{Rh}\right)$. The onset of the choroidal nonperfusion was dose dependent, occurring earlier with higher doses of radiation. Whilst choroidal atrophy occurred in some areas, some of the residual choroidal vasculature was stained with fluorescein dye. ${ }^{9}$ However, FFA visualisation of the choroid clinically and experimentally is not ideal and is only optimum where RPE and choriocapillaris atrophy are significant.

Indocyanine green angiography (ICG-A), which permits better visualisation of the choroidal vasculature in vivo (compared with FFA), has recently been introduced into clinical use. ${ }^{10,11}$ It allows visualisation of the choroidal vasculature even in the presence of moderate lens opacities. The purpose of this study is to describe the ICG-A changes of radiation choroidopathy and to relate them to the natural course of the disease.

We reviewed the ICG-A changes in the choroidal vasculature in the irradiated ('normal') peri-tumoral zone in 16 patients between 4 to 72 months following ${ }^{106} \mathrm{Ru} /{ }^{106} \mathrm{Rh}$ brachytherapy for choroidal melanoma.

\section{MATERIALS AND METHODS}

Patients included in this study had choroidal melanoma treated conservatively with local irradiation from ${ }^{106} \mathrm{Ru} /{ }^{106} \mathrm{Rh}$ sources in our clinic. The plaques used are similar to those described by Lommatzsch $^{3,6}$ and deliver a dose of $100 \mathrm{~Gy}$ to the tumour apex (800-1000 Gy to tumour base/sclera). ${ }^{12}$

All patients had funduscopy, fundus photography, 
FFA (on a Topcon TRC 50VT camera) and ultrasonography before treatment as well as during their follow-up. The patients included in this study also had ICG-A at different time intervals following treatment (usually on the same day as FFA) as part of their follow-up.

In an initial period between April 1986 and September 1988, a Topcon TRC 50VT fundus camera equipped with a continuous power light source from a 300 watt halogen bulb and an infraredsensitive camera connected to a video recorder were used. In the second period from July 1992 to December 1993, a Topcon Imagenet Digital Imaging System linked to a Topcon 50IA camera specially designed for ICG-A and FFA $^{13}$ was available for the study.

For the ICG-A procedure, $25 \mathrm{mg}$ ICG was dissolved in $1 \mathrm{ml}$ aqueous solvent and injected into the antecubital vein followed by a $5 \mathrm{ml}$ aqueous solvent flush through a three-way tap. In addition, during the second period of the study, images were

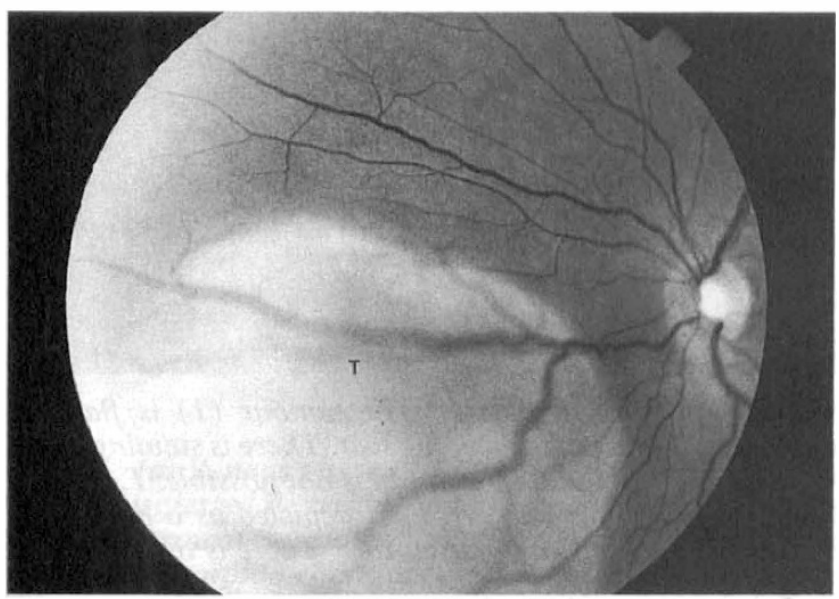

(a) obtained at approximately 5 second intervals for the first minute then every 5 minutes for at least 20 minutes. The early phases of the procedure were simultaneously recorded on videotape. After completion of the examination, the images were reviewed and downloaded to an optical disc for storage and analysis.

\section{RESULTS}

A total of 16 patients treated with ${ }^{106} \mathrm{Ru} /{ }^{106} \mathrm{Rh}$ brachytherapy are included in this study. All the eyes showed tumour regression clinically and echographically. Six of these eyes had ICG-A done at 4-6 months, and 8 (including 4 re-examined from the 4-6 month group) had ICG-A at 10-24 months following treatment. Six others had multiple examinations between 30 and 72 months after brachytherapy.

ICG-A clearly demonstrated the extent of the degenerative changes in the choroidal vasculature. There were varying degrees of alteration in the

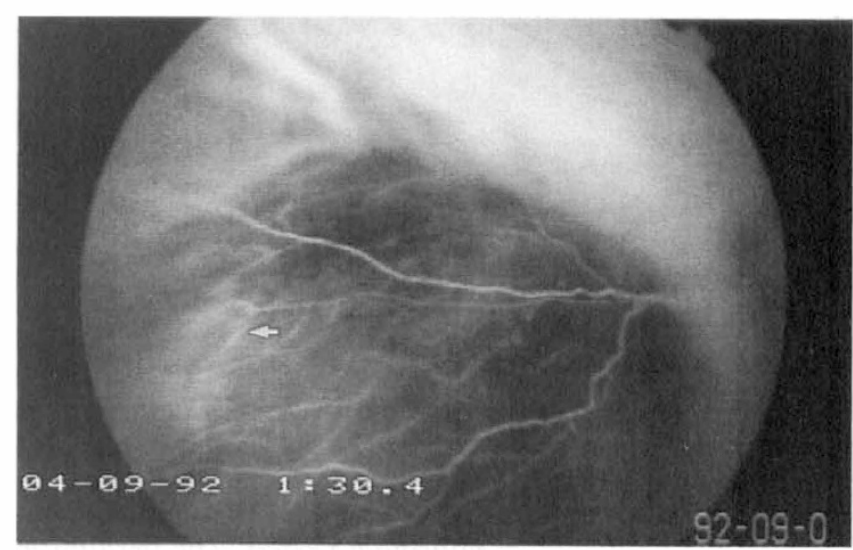

(b)

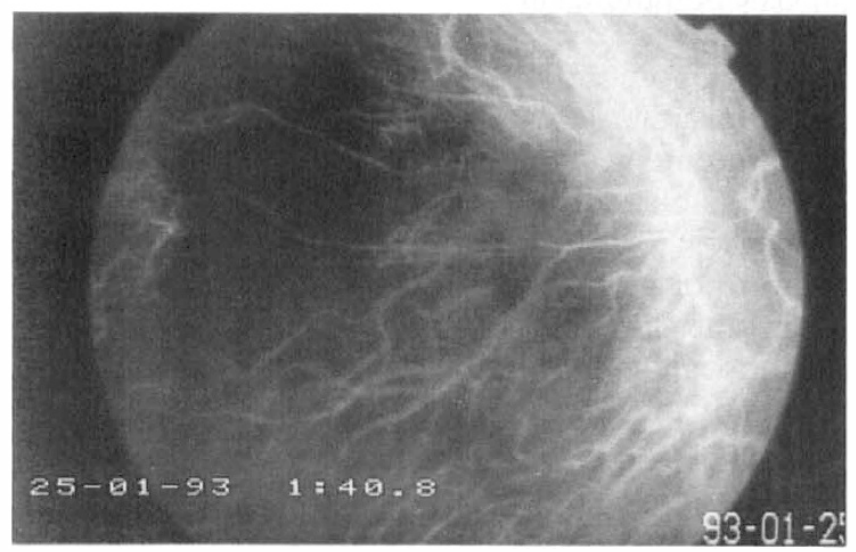

(c)

Fig. 1. (a) Red-free fundus photograph of choroidal melanoma. The tumour mass (T) displaces the overlying retinal vasculature. (b) and (c) ICG-A of choroidal melanoma in the same eye. (b) Pre-treatment angiography shows abnormal intratumoral choroidal vascularisation. The irregular branching vasculature is associated with local dye leak in the late angiographic phases (arrow). (c) Four months after radiotherapy. There is peritumoral small vascular closure. In addition there is reduction in tumour dye leak compared with that observed in the pre-treatment angiograms. 


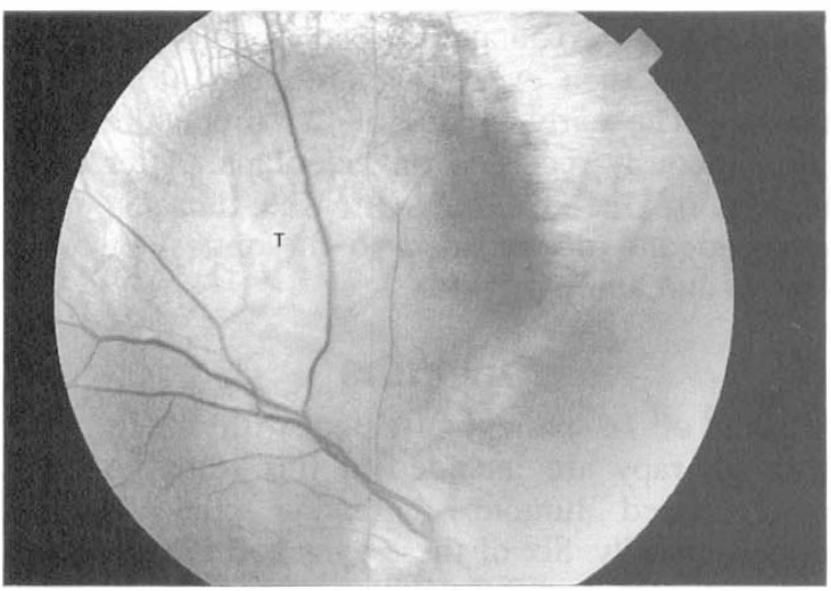

(a)

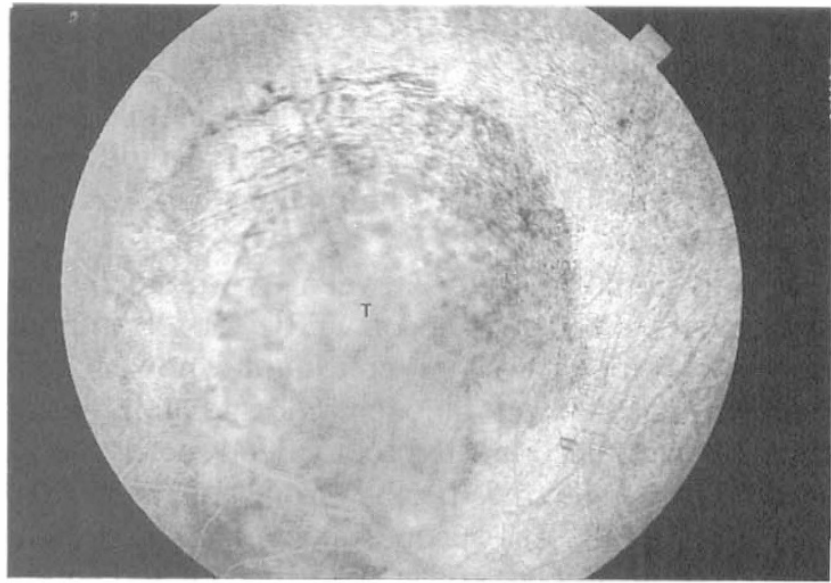

(b)

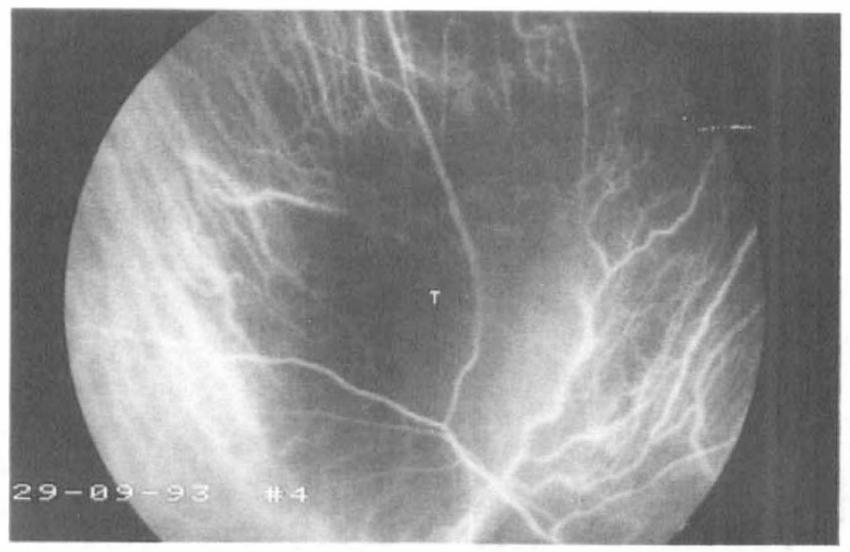

(c)

Fig. 2. (a) Red-free photograph of choroidal melanoma 21 months after brachytherapy. The tumour (T) is flat and surrounded by an atrophic zone. (b) Late venous phase FFA of the same eye showing an atrophic scar. There is staining of the sclera seen through atrophic RPE and choriocapillaris. Detailed evaluation of surrounding choroid is not possible. T, tumour scar. (c) ICG-A of choroidal melanoma 21 months after brachytherapy. The tumour scar (T) is depicted as a dark zone crossed only by the larger choroidal vessels. The peritumoral area shows significant vascular closure. There is only minimal staining of the vascular wall and no dye leak is observed.

architecture of the choroidal microcirculation, capillary incompetence and closure.

The larger choroidal vessels seemed more resistant to radiation injury than the choriocapillaries and smaller choroidal arterioles and venules. Single enlarged choroidal vessels became visible with time and their walls stained with ICG dye.

Similar angiographic changes were observed with FFA, but were less obvious in the absence of significant RPE atrophy. The choriocapillary filling with FFA was more visible in the early angiographic phases in some patients when compared with ICG visualisation.

\section{From 4 to 6 Months}

The earliest ICG-A changes observed were peritumoral atrophy of RPE and irregular closure of choriocapillaries and some smaller arterioles and venules (Fig. 1). Most of the larger choroidal vessels remained perfused at this stage. Occasionally, the large choroidal vessels showed slight dilatation. There was no evidence of ICG dye staining of choroidal vascular walls in the peritumoral area.

\section{From 10 to 24 Months}

The irregular choroidal vascular occlusion, especially of the small-calibre vessels, was now more noticeable in the normal peritumoral choroid than it had been at 4-6 months (Fig. 2). The residual vessels were of irregular calibre and showed minimal localised dye staining of their walls.

\section{From 30 to 72 Months}

ICG-A confirmed the atrophic changes seen on FFA in the non-tumour treated chorioretina. Only the larger choroidal vessels persisted, with remarkable calibre variation of beading and telangiectatic-like dilatations (Figs. 3-6). There was significant beading, especially of the larger arterioles. Focal outpouchings similar to microaneurysms observed in the retinal 


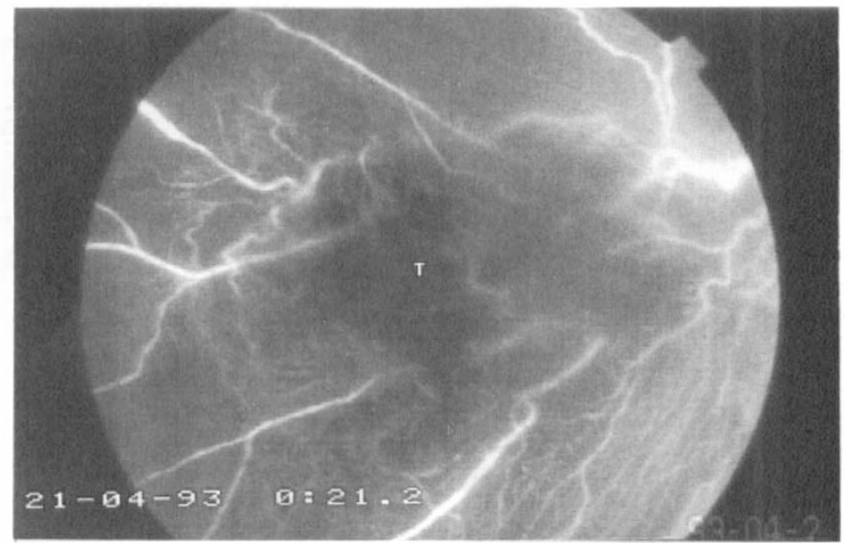

Fig. 3. ICG-A of choroidal melanoma 5 years after brachytherapy. There is extensive irregular vascular closure around the tumour scar $(T)$. The residual large choroidal vessels are irregular in calibre, stain irregularly with dye and leak locally. Dilated choroidal vessels are observed at the nasal edge of the tumour scar.

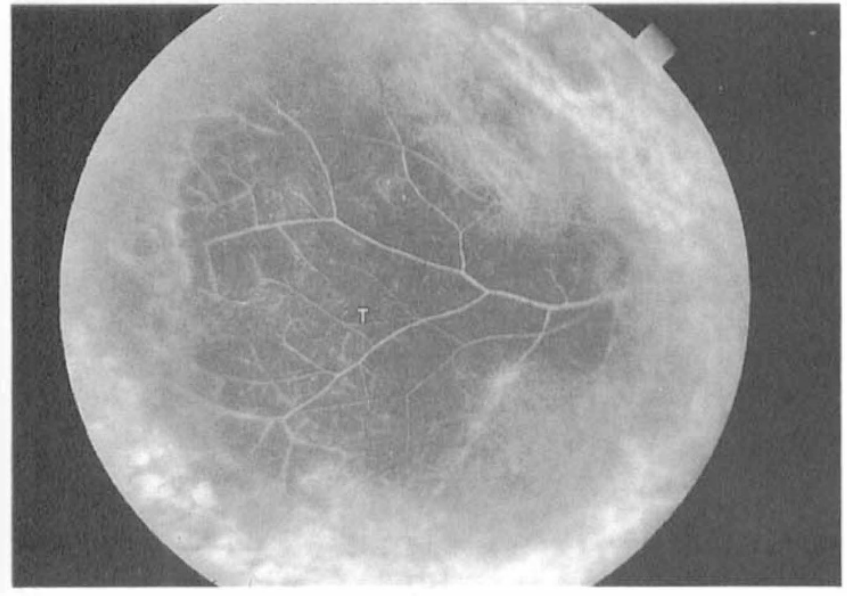

(a)

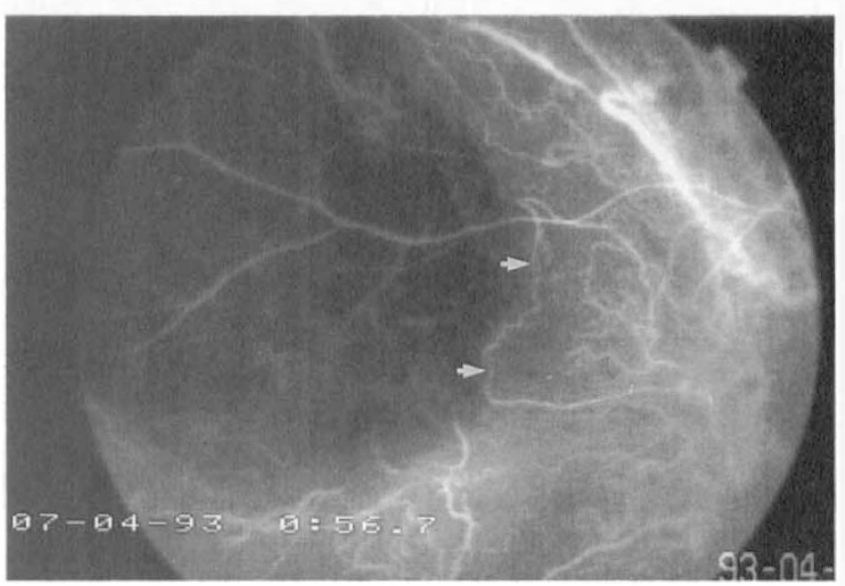

(b)

Fig. 4. (a) Venous phase FFA of choroidal melanoma 3 years after brachytherapy. The tumour scar $(T)$ is depicted as a dark area transgressed by residual retinal vasculature. The surrounding atrophic (irradiated) zone stains with fluorescein. No details of the choroidal circulation are discernible. (b) Early phase ICG-A of choroidal melanoma 3 years after brachytherapy. A presumed choroido-choroidal vascular anastomosis (arrows) is seen at the edge of the hypofluorescent zone. Extensive vascular closure is observed. There is staining of the residual choroidal vessels, which are of irregular calibre and show focal dilatations.

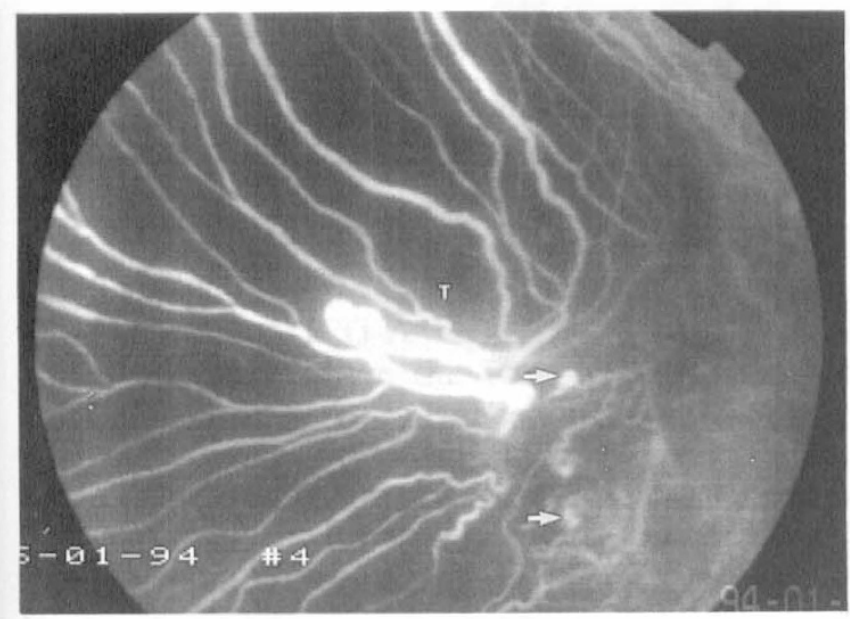

(a)

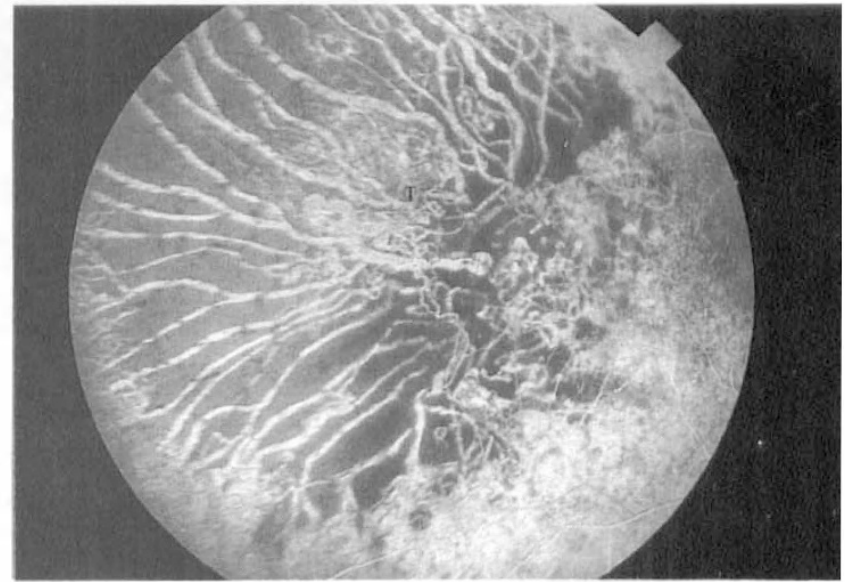

(b)

Fig. 5. (a) ICG-A of choroidal melanoma $4 \frac{1}{2}$ years following plaque treatment. Extensive small vessel closure is noted after brachytherapy. Only the larger choroidal vessels are seen transgressing the irradiated zone. There is irregular calibre variation and staining of the walls with dye and microaneurysm formation (arrows). T, tumour scar. (b) Venous phase FFA of same eye as in (a). The extensive choroidal vascular closure is observed through atrophic RPE. Microaneurysms are visible but less distinct. T, tumour scar. 


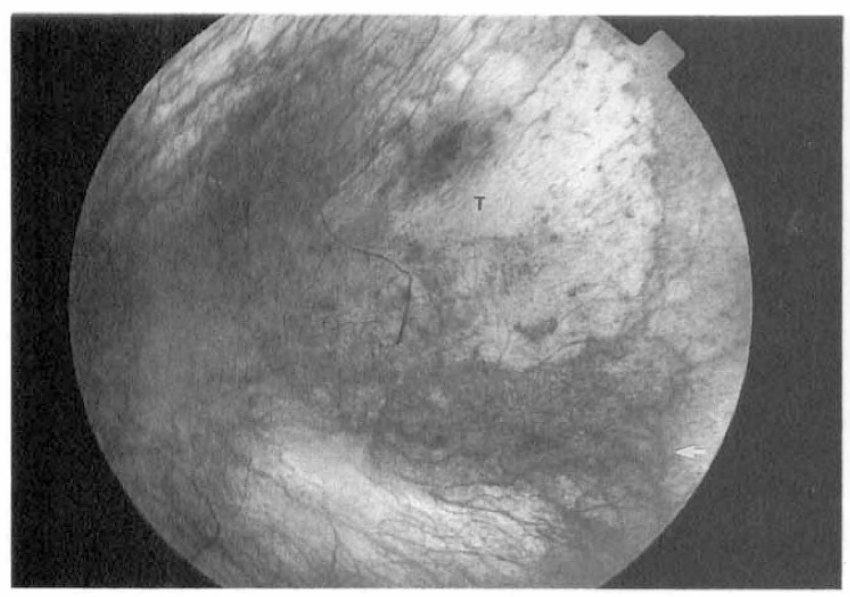

(a)

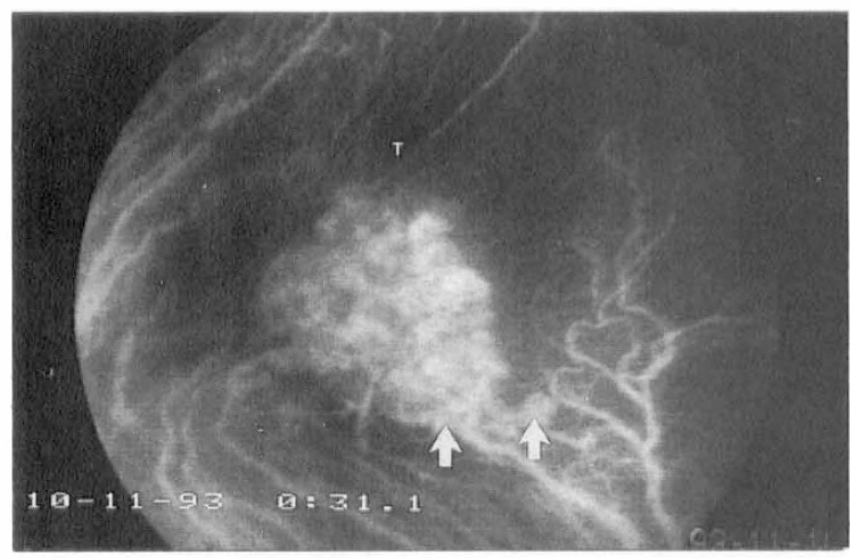

(c)

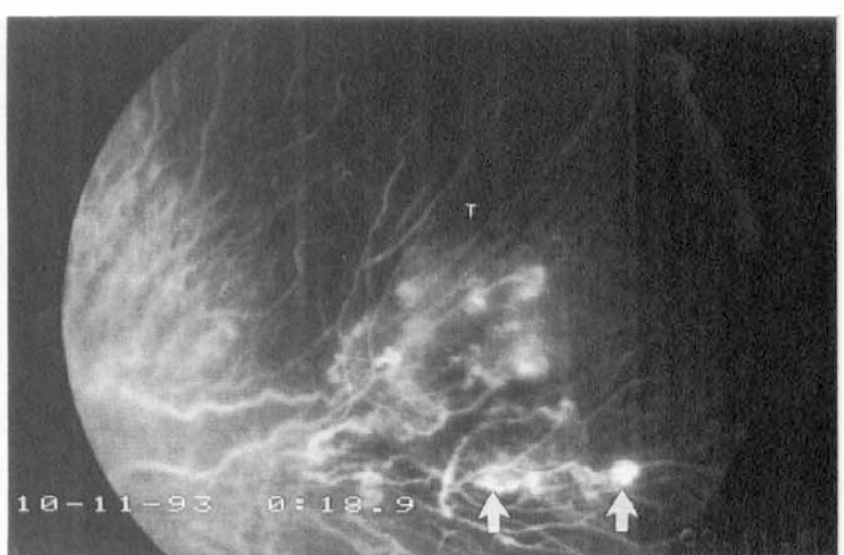

(b)

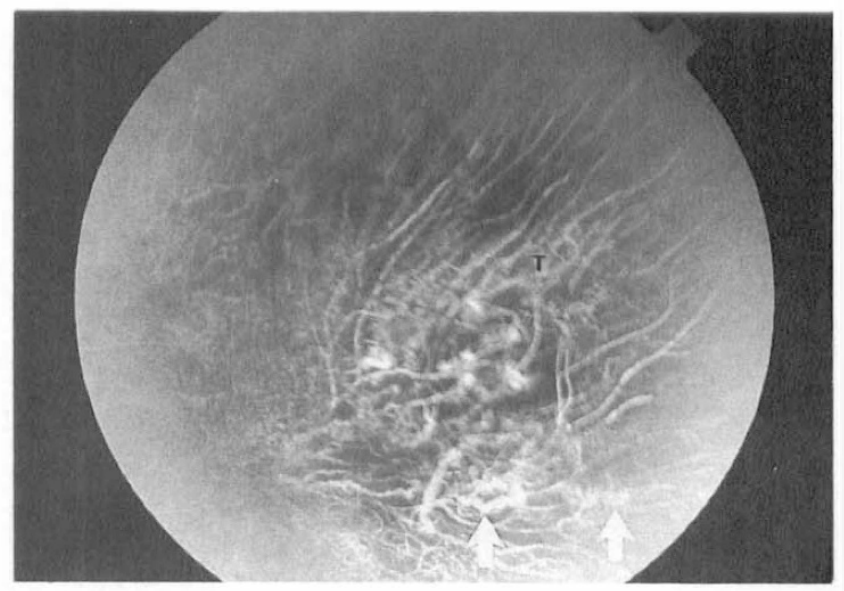

(d)

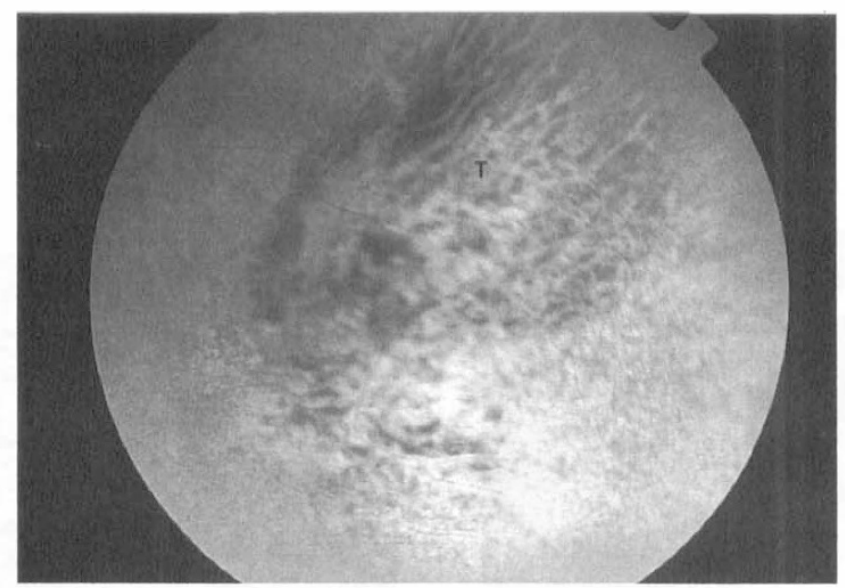

(e)

Fig. 6. (a) Red-free photograph of choroidal melanoma 52 months following brachytherapy. The tumour scar (T) is flat. There is irregular retinal vascular closure. Some of the residual large choroidal vessels are visible through atrophic RPE (arrow). (b) and (c) ICG-A of regressed choroidal melanoma 52 months after brachytherapy. Irregular choroidal vascular closure is obvious all round the atrophic tumour scar $(T)$. Vascular beading, focal dilatations or microaneurysm are noted at the borders of vascular closure. Choroidal neovascular vessels (arrows) which leak profusely are observed inferiorly outside the tumour scar $(T)$. These new vessels progressively leaked dye into the surrounding tissue and are drained by efferent vessels which run directly into the vortex ampulla inferiorly. (d) and (e) FFA of the same eye in (a) performed on the same day. The choroidal vascular closure is less obvious (cf. ICG-A). The choroidal neovascularisation is not as clearly depicted as with ICG-A. T, tumour scar. Arrows correspond to the same structures arrowed in Fig. $6(b)$. 
vasculature were observed. Some of these vessels, including the microaneurysms, leaked dye locally. Residual vessels showed dilatation at the edge of ischaemic zones (Fig. 3).

There was progressive staining of the larger choroidal vessels: 'perivasculitis' (Figs. 3-6). This was associated with progressive closure of the lumen of these vessels. Such vascular staining was not observed earlier than 2 years following radiation treatment, and was very marked in all eyes examined ( $>2$ years).

Vascular anastomoses, which were presumed choroido-choroidal, were detected at the edges of some of the atrophic scars (Fig. 4). In some eyes, new clusters of abnormal choroidal vessels were observed more than 2 years following treatment. In one case, focal choroidal new vessels were observed with ICG (Fig. 6) outside the original tumour area. These new vessels leaked ICG dye profusely, but were less demonstrable with fluorescein angiography. These choroidal neovascular malformations were different in location and morphology from the intratumoral vascularisation observed in untreated melanoma. Furthermore echography did not show any evidence of tumour recurrence. The choroidal new vessels when observed over a period of 12-18 months increased in size in the absence of echographic tumour recurrence.

\section{DISCUSSION}

Histological findings in the choroid from eyes treated with radiotherapy for choroid melanomas are similar irrespective of the source of radiation. ${ }^{8}$ These changes include sclerosis of choroidal vessels, atrophy of the different layers of the choroid and Bruch's membrane, and proliferation of the RPE. ${ }^{14}$

FFA has also been used simultaneously to assess the chorioretina for complications of radiotherapy, and follow-up of tumour regression/involution. ${ }^{5,15-17}$ The reported changes in the choroid were choroidal telangiectasis ${ }^{15,16}$ and progressive vascular closure, but the smaller choroidal vessels were visible only where the RPE atrophy and partial/total loss of the choriocapillaries were sufficiently advanced. ${ }^{5}$

In this study, ICG-A highlighted the choroidal circulation even where the RPE atrophic changes were not present or advanced enough to allow adequate view of the choroidal vasculature with FFA. The angiograms demonstrated that morphologically the features of radiation choroidal vasculopathy - beading or irregular calibre variation, telangiectatic-like dilatations, microaneurysms and new vessel formation - were similar to those previously described in the retina. This ICG-A study further confirmed that vascular closure occurred in the peritumoral normal choroid within 3-4 months of brachytherapy. The vaso-occlusion seemed to occur in the smaller vessels initially and slowly progressed to involve the larger vessels, i.e. the rate and degree of choroidal vascular closure seemed to be inversely related to the size of the vessels. Similar patterns have been noted in retinal vascular response to radiation injury. ${ }^{18-20}$ These findings are consistent with those of Hayreh ${ }^{15}$ and McFaul and Bedford, ${ }^{16}$ who also showed telangiectasis of the residual choroidal vessels in radiation choroidopathy following brachytherapy for choroidal melanoma.

Vascular wall staining was demonstrable only in the larger choroidal vessels and was observed in the late post-radiation period. Some of the vessels which stained subsequently became non-perfused in the longer term after radiotherapy. Furthermore, staining of vascular walls observed angiographically (with FFA in previous studies and ICG-A in this study) always seemed to be associated with occlusion of the adjoining vessels or the particular vessels at a later date. Vascular wall staining, however, did not necessarily precede all vascular occlusions.

It has been suggested by some authors ${ }^{17,21}$ that inflammation of the choroid occurs in the early postirradiation period and that this inflammation leads to fluorescein dye leak into the vitreous in the early post-treatment period, and staining of individual vascular walls later. ${ }^{9}$ However, despite histopathological demonstrations of early choroidal oedema and subsequent atrophy and occlusion of choroidal vasculature (in experimental and clinical studies) no evidence of a true vasculitis has ever been demonstrated in any of these studies. ${ }^{5,9-17}$

It is suggested that choroidal vascular wall staining with ICG in radiation choroidopathy is due to radiation-induced endothelial cell loss and/or dysfunction rather than a true vasculitis. Progression leads to vascular occlusion. This thesis is consistent with previous observation of platelet aggregates in the choriocapillaries in experimental studies of radiation choroidopathy. ${ }^{22}$

Of particular interest was the observation of choroidal neovascularisation in the irradiated zone outside the original tumour area. These new vessels were more distinctly visualised with ICGA than on FFA. The choroidal new vessels observed in our patient were not thought to represent tumour recurrence since funduscopy and echography demonstrated a flat scar, and there was no evidence of tumour regrowth. Furthermore, the new vessels were located outside the original tumour area, and the ICGA morphology of these vessels was different from that observed in untreated or inadequately treated melanomas.

Choroidal neovascularisation is occasionally associated with untreated choroidal melanomas ${ }^{23}$ and has also been reported after successful treatment of 
choroidal melanomas with photocoagulation ${ }^{24,25}$ and following brachytherapy for retinoblastoma. ${ }^{26}$ To our knowledge there are no previous reports in the literature of choroidal neovascularisation associated with irradiated melanoma. The pathophysiological mechanisms responsible for the choroidal neovascularisation following radiation injury are possibly related to those that occur in the retina, as well as to the disorganisation of the outer retina and Bruch's membrane produced by the very high doses of ionising radiation. However, since sizeable choroidal melanomas have pronounced effects on the involved and surrounding retinal and choroidal vasculature, it may be inaccurate to attribute the neovascularisation to the effects of ionising radiation alone.

The presumed choroido-choroidal vascular channels filled rapidly from the choroidal circulation and remained competent, whereas the choroidal new vessels leaked dye. These channels represent a component of the healing process similar to the chorioretinal vascular anastomoses described within chorioretinal scars. ${ }^{27}$ Alternatively, they may represent dilatation of pre-existing vascular channels following occlusion of the more distal choroidal arteriolar circulation.

We postulate that the mechanism of radiation vasculopathy in the choroid is similar to that in the retina. The initiating event is localised endothelial cell loss which precipitates a cascade of events producing vascular occlusions. ${ }^{20,22}$

This study is supported by a grant from 'Les Amis des Aveugles', Gent, Belgium.

Key words: Brachytherapy, Choroidal melanoma, Choroidal vasculopathy, Indocyanine green angiography (ICG-A), Neovascularisation, Radiation choroidopathy.

\section{REFERENCES}

1. McFaul PA. Local radiotherapy in the treatment of malignant melanoma of the choroid. Trans Ophthalmol Soc UK 1977;97:421-7.

2. Wessing A, Foerster M, Fried M. Fluoresceinzangiografische Befunde nach Ruthenium-Behandlung maligner Aderhautmelanome. Fortschr Ophthalmol 1983;80:415-7.

3. Lommatzsch PK. ß-irradiation of choroidal melanomas with $\mathrm{Ru}^{106} / \mathrm{Rh}^{106}$ applicators: 16 years' experience. Arch Ophthalmol 1983;101:713-7.

4. Foerster $\mathrm{MH}$, Burnfield $\mathrm{N}$, Wessing $\mathrm{A}$, et al. Die Behandlung von malignen Melanome der Uvea mit 106-Ruthenium-Applikatoren. Klin Monatsbl Augenheilkd 1984;85:490-4.

5. Tarkkanen A, Laatikainen L. Fluorescein angiography in the long-term follow-up of choroidal melanoma after conservative treatment. Acta Ophthalmol (Copenh) 1985;63:73-9.

6. Lommatzsch PK. Results after ß-irradiation of choroidal melanomas: 20 years' experience. Br J Ophthalmol 1986;70:844-851.

7. Seddon JM, Gragoudas ES, Albert DM. Ciliary body and choroidal melanomas treated by proton beam irradiation: histopathologic study. Arch Ophthalmol 1983;101:1402-8.

8. Messner E, Bornfield N, Foerster M, et al: Histopathological findings in eyes treated with ruthenium plaque for uveal melanoma. Graefes Arch Clin Exp Ophthalmol 1992;230:391-6.

9. Schaling DF, Lommatzsch PK, van Delft JL, et al. Effect of beta-irradiation by a 106 ruthenium plaque on the rabbit eye choroid. Graefes Arch Clin Exp Ophthalmol 1989;227:194-9.

10. Bischoff PM, Flower RW. Ten years' experience with choroidal angiography using indocyanine green dye: a new routine examination or an epilogue? Doc Ophthalmol 1985;60:235-91.

11. Hayashi K, De Laey JJ. Indocyanine green angiography of submacular choroidal vessels in the human eye. Ophthalmologica 1985;190:20-9.

12. Van Hijfte R, Verbraeken H, De Laey JJ. Ruthenium applicators in the treatment of choroidal melanomas. Bull Soc Belge Ophthalmol 1987;224:51-8.

13. Yannuzi LA, Slakter JS, Sorenson JA, et al. Digital indocyanine green videoangiography and choroidal neovascularisation. Retina 1992;12:191-223.

14. McFaul PA, Morgan J. Histopathological changes in malignant melanomas of the choroid after cobalt therapy. Br J Ophthalmol 1977;61:221-8.

15. Hayreh SS. Postradiation retinopathy: a fluorescence fundus angiographic study. $\mathrm{Br} \mathrm{J}$ Ophthalmol 1970;54:705-14.

16. McFaul PA, Bedford MA. Ocular complications after therapeutic irradiation. Br J Ophthalmol 1970;54:23747.

17. Lommatzsch PK, Ballin RE, Helm W. Fluorescein angiography in the follow-up study of choroidal melanoma after ${ }^{106} \mathrm{Ru} / 7^{106} \mathrm{Rh}$ plaque therapy. Retina 1987;7:148-55.

18. Amoaku WMK, Archer DB. Cephalic radiation and retinal vasculopathy. Eye 1990;4:194-203.

19. Amoaku WMK, Archer DB. Fluorescein angiographic features: natural course and treatment of radiation retinopathy. Eye 1991;4:657-67.

20. Archer DB, Amoaku WMK, Gardiner TA. Radiation retinopathy: clinical, histopathological, ultrastructural and experimental correlations. Eye 1991;5:239-57.

21. Lommatzsch PK. Morphologische and funktionelle Veranderungen des Kaninchenauges nach Einwirkung von Betastrahlen $\left({ }^{106} \mathrm{Ru} /{ }^{106} \mathrm{Rh}\right)$ auf den dorsalen Bulbusabschnitt. Graefes Arch Clin Exp Ophthalmol 1968;176:100-25.

22. Amoaku WMK, Mahon G, Gardiner TA, Frew L, Archer DB. Late ultrastructural changes in the rat retina following low-dose $\mathrm{x}$-irradiation. Graefes Arch Clin Exp Ophthalmol 1992;230:659-74.

23. Shields JA, Shields CL. Intraocular tumours: a text and atlas. Philadelphia: WB Saunders, 1992.

24. Gerke E, Mackensen D, Burnfeld N. Neovaskularisationen als Komplikation bei der Lichtkoagulation maligner Aderhautmelanome. Ber Dtsch Ophthalmol Ges 1981;78:525-7.

25. Augsberger JJ, Piro RA, Sheilds JA, et al. Scatter photocoagulation for chorioretinal neovascularisation. Retina 1984;4:171-6.

26. Archer DB, Amoaku WMK, Kelly G. Chorioretinal neovascularisation following radon seed treatment of retinoblastoma in 2 patients. $\mathrm{Br} \mathrm{J}$ Ophthalmol 1993;777:95-9.

27. Green WR, Key SN III. Senile macular degeneration: a histological study. Trans Am Ophthalmol Soc 1977;75:180-254. 